\title{
Effect of Yoga on Cardio Respiratory Parameter in Medical Students
}

\author{
Ashok Solanki ${ }^{1}$, Dharmistha Dodiya ${ }^{2}$, Chandan Chakravarti ${ }^{3}$, Jaydeep Kagathara ${ }^{4}$ \\ ${ }^{1}$ Associate Professor, Dept. of Physiology, Smt. NHL Municipal Medical College, Ahmedabad, ${ }^{2}$ Tutor, Dept. of \\ Physiology, GMERS Medical College,, Junagadh, ${ }^{3}$ Professor, Dept. of Biochemistry, Smt. NHL Municipal Medical \\ College, Ahmedabad, ${ }^{4}$ Associate Professor, Dept. of Physiology, GCS Medical College Hospital \& Research \\ Centre, Ahmedabad
}

\begin{abstract}
The competition in medical field, unhealthy food and lack of exercise deteriorate the health of student. Yoga is the ancient technique to increase the concentration power, physical and mental well-being. We have done randomized control study on medical students between study and control group. The study group has practice yoga for 4 weeks whereas the control group hasn't. In the study group there is significantly improvement in cardio-respiratory parameters $(\mathrm{p}<0.0001)$. This study showed that regular yoga practice in medical students can decrease their stress and improve cardiac and respiratory function.
\end{abstract}

Keywords: Yoga, stress, medical students, cardio-respiratory parameters.

\section{Introduction}

Medical students are confronted with a variety of life stressors from both college and home. The disproportion between sympathetic and parasympathetic discharge due to stress is associated with morbid condition like diabetes mellitus, hypertension and metabolic syndrome ${ }^{(1)}$.

Yoga is development of the union of mind and body through a combination of exercise respiration and meditation in order to achieve psychosomatic harmony ${ }^{(2)}$. Yogaconsists of a holistic combination of postural exercise (Asana) ${ }^{(3)}$, relaxation and voluntary breathing exercise (pranayamas). The yoga has been studied in patient of diabetes, hypertension and post traumatic stress disorder for management ${ }^{(4)(5)(6)}$.

Hence it is pertinent to study of the effects of yoga on cardio respiratory physiology of medical students

\section{Corresponding Author:}

\section{Dr. Dharmistha Dodiya}

Tutor, Dept. of Physiology, GMERS Medical College,, Junagadh

Mobile: 8866506544

e-mail: dodiyadharmishtha@gmail.com in order to better understand its effects on healthy individuals and to provide the scientific basis for the possible use of yoga techniques as preventive and/or alternative therapy for health disorders ${ }^{(7)(8)}$.

\section{Material and Method}

Study Design - Randomized control study

\section{Inclusion criteria:}

1. Those who were ready to give written consent.

2. Those who were above 18 years.

3. Those who were apparently healthy on history and clinical examination.

\section{Exclusion criteria:}

1. Those who had BMI $<18.5$ or $>24.99$

2. Those who were on any diet/exercise regime.

3. Those who were on any drugs that affect cardiorespiratory functions.

4. Those who had any disease/disorder that can affect physiological functions e.g. thyroid disorders, diabetes mellitus bronchial asthma any acute illness of respiratory or cardiovascular system. 
5. Those who were smokers and/or alcoholics.

Sample size- 300

Randomization: Using simple random sampling, participants were allotted to intervention study group and control group.

\section{Intervention:}

- Four weeks yoga training program by a certified yoga teacher.

- Program included yoga training for 6 days a week with one-day rest.

- Graded yoga training schedule with weekly medical examination of subjects.

\begin{tabular}{|c|l|c|}
\hline Week No. & Yoga training & Total daily duration \\
\hline 1 & Joint relaxation exercise(JRE) for 15 minutes followed by shavasan for 5 minutes & $15+5=20$ minutes \\
\hline Weekly medical examination - 1 & $5+10+5=20$ minutes \\
\hline 2 & $\begin{array}{l}\text { JREs for } 5 \text { minutes followed by pranayams for } 10 \text { minutes followed by shavasan for } 5 \\
\text { minutes. }\end{array}$ & $5+10+5+5=25$ minutes \\
\hline Weekly medical examination - 2 & $\begin{array}{l}\text { JREs for } 5 \text { minutes followed by pranayams for } 10 \text { minutes followed by suryanamaskar for } 5 \\
\text { minutes followed by shavasan for } 5 \text { minutes. }\end{array}$ & $5+10+10+5=30$ minutes \\
\hline 3 & $\begin{array}{l}\text { JREs for } 5 \text { minutes followed by pranayams for } 10 \text { minutes followed by suryanamaskar for } \\
10 \text { minutes followed by shvasan for } 5 \text { minutes }\end{array}$ \\
\hline Weekly medical examination - 3 &
\end{tabular}

\section{Pranayam included following:}

1. Anulomvilom pranayama

2. Bhramari pranayama

3. Shitali pranayama

4. Sitkari pranayama

General Parameters studied were Height, Weight and Body Mass Index. The cardiac parameter studied were Resting Pulse Rate (RPR), Resting Systolic and Diastolic Blood Pressure and Fitness Index (FI).

Fitness index: FI was calculated using the Harvard step test ${ }^{(9)}$.

FI $(\%)=(100 \times$ test duration in seconds $)$ divided by ( $2 \mathrm{x}$ sum of heart beats in the recovery periods)

The Respiratory Parameters were measured using a computerized spirometer. The tests recorded were tidal volume (TV), slow vital capacity (SVC), forced vital capacity (FVC), forced expiratory volume in first second
(FEV1), FEV/FVC ratio, Peak expiratory flow rate (PEFR), forced expiratory flow during middle 50\% (FEF 25-75\%),Forced expiratory flow during 200-1200ml of expiration $\left(\mathrm{FEF}_{200-1200}\right)$ and maximum voluntary ventilation (MVV). The respiratory parameters for efficiency measured were Breatheholding (BHT), Maximum expiratory pressure (MEP) and $40 \mathrm{~mm} \mathrm{Hg}$ endurance test (40mmHg ET).

\section{Statistical analysis:}

To compare a mean of different variables each group i.e. intervention and control group (post-test value versus pre-test value), paired students test was used.

To compare mean of different variables between the groups i.e. intervention and control group unpaired students test was used.

The alpha level to determine significance was $\mathrm{P}<0.05$ for all analysis. $\mathrm{p}<0.05$ shall be considered highly significant. 
Findings:

Table 1: Anthropometric parameters in intervention group (paired student's test)

\begin{tabular}{|c|c|c|c|c|}
\hline \multirow{2}{*}{ Gender } & \multirow{2}{*}{ Parameter } & Pre yoga & Post yoga & \multirow{2}{*}{$P$ value } \\
\hline & & $\operatorname{Mean} \pm$ SD & Mean \pm SD & \\
\hline \multirow{4}{*}{$\begin{array}{l}\text { Female } \\
(\mathrm{n}=72)\end{array}$} & Age(years) & $18.50 \pm 0.71$ & $18.50 \pm 0.71$ & - \\
\hline & Height(cm) & $156.46 \pm 5.29$ & $156.46 \pm 5.29$ & - \\
\hline & Weight(kg) & $52.00 \pm 6.44$ & $52.03 \pm 6.39$ & 0.32 \\
\hline & $\mathrm{BMI}(\mathrm{Kg} / \mathrm{m} 2)$ & $21.22 \pm 2.26$ & $21.24 \pm 2.25$ & 0.39 \\
\hline \multirow{4}{*}{$\begin{array}{l}\text { Male } \\
(\mathrm{n}=78)\end{array}$} & Age(years) & $18.60 \pm 0.62$ & $18.60 \pm 0.62$ & - \\
\hline & Height $(\mathrm{cm})$ & $170.72 \pm 7.64$ & $170.72 \pm 7.64$ & - \\
\hline & Weight(kg) & $61.69 \pm 8.81$ & $61.64 \pm 8.54$ & 0.44 \\
\hline & $\operatorname{BMI}(\mathrm{Kg} / \mathrm{m} 2)$ & $21.14 \pm 2.46$ & $21.12 \pm 2.37$ & 0.47 \\
\hline \multirow{4}{*}{$\begin{array}{l}\text { All } \\
(n=150)\end{array}$} & Age(years) & $18.43 \pm 0.67$ & $18.43 \pm 0.67$ & - \\
\hline & Height $(\mathrm{cm})$ & $163.87 \pm 9.73$ & $163.87 \pm 9.73$ & - \\
\hline & Weight(kg) & $57.04 \pm 9.14$ & $57.03 \pm 8.96$ & 0.72 \\
\hline & BMI(Kg/m2) & $21.18 \pm 2.36$ & $21.18 \pm 2.31$ & 0.86 \\
\hline
\end{tabular}

Table 2: Anthropometric parameters in control group (paired student's test)

\begin{tabular}{|c|c|c|c|c|}
\hline \multirow{2}{*}{ Gender } & \multirow{2}{*}{ Parameter } & Pre yoga & Post yoga & \multirow{2}{*}{$P$ value } \\
\hline & & Mean \pm SD & Mean \pm SD & \\
\hline \multirow{4}{*}{$\begin{array}{l}\text { Female } \\
(n=64)\end{array}$} & Age(years) & $18.09 \pm 0.29$ & $18.09 \pm 0.29$ & - \\
\hline & Height(cm) & $158.94 \pm 5.51$ & $158.94 \pm 5.51$ & - \\
\hline & Weight(kg) & $53.16 \pm 6.44$ & $53.13 \pm 7.22$ & 0.57 \\
\hline & $\mathrm{BMI}(\mathrm{Kg} / \mathrm{m} 2)$ & $20.97 \pm 2.04$ & $20.96 \pm 2.01$ & 0.62 \\
\hline \multirow{4}{*}{$\begin{array}{l}\text { Male } \\
(\mathrm{n}=86)\end{array}$} & Age(years) & $18.23 \pm 0.48$ & $18.23 \pm 0.48$ & - \\
\hline & Height $(\mathrm{cm})$ & $171.00 \pm 6.43$ & $171.00 \pm 6.43$ & - \\
\hline & Weight(kg) & $62.20 \pm 8.64$ & $62.13 \pm 8.50$ & 0.13 \\
\hline & $\mathrm{BMI}(\mathrm{Kg} / \mathrm{m} 2)$ & $21.22 \pm 2.23$ & $21.20 \pm 2.19$ & 0.15 \\
\hline \multirow{4}{*}{$\begin{array}{l}\text { All } \\
(n=150)\end{array}$} & Age(years) & $18.17 \pm 0.41$ & $18.17 \pm 0.41$ & - \\
\hline & Height(cm) & $165.85 \pm 8.50$ & $165.85 \pm 8.50$ & - \\
\hline & Weight(kg) & $58.34 \pm 9.23$ & $58.29 \pm 9.13$ & 0.13 \\
\hline & $\mathrm{BMI}(\mathrm{Kg} / \mathrm{m} 2)$ & $21.11 \pm 2.15$ & $21.10 \pm 2.11$ & 0.17 \\
\hline
\end{tabular}

Table 3: Cardiovascular efficiency in intervention group (paired student's test)

\begin{tabular}{|l|l|c|c|c|}
\hline \multirow{3}{*}{ Gender } & \multirow{2}{*}{ Parameter } & Pre yoga & Post yoga & \multirow{2}{*}{ P value } \\
\cline { 2 - 5 } & & Mean \pm SD & Mean \pm SD & $<0.01$ \\
\hline \multirow{3}{*}{$\begin{array}{l}\text { Female } \\
(\mathrm{n}=72)\end{array}$} & RPR(beats/min) & $75.31 \pm 5.39$ & $73.04 \pm 5.26$ & $<0.01$ \\
\cline { 2 - 5 } & SBP(mmHg) & $116.75 \pm 8.68$ & $114.44 \pm 5.43$ & $<0.01$ \\
\cline { 2 - 5 } & DBP(mmHg) & $75.95 \pm 4.28$ & $74.06 \pm 4.49$ & $<0.01$ \\
\cline { 2 - 5 } & $\mathrm{fI}(\%)$ & $69.93 \pm 12.10$ & $71.31 \pm 10.34$ & $<$ \\
\hline
\end{tabular}




\begin{tabular}{|c|c|c|c|c|}
\hline \multirow{2}{*}{ Gender } & \multirow{2}{*}{ Parameter } & Pre yoga & Post yoga & \multirow{2}{*}{$P$ value } \\
\hline & & $\operatorname{Mean} \pm$ SD & Mean \pm SD & \\
\hline \multirow{4}{*}{$\begin{array}{l}\text { Male } \\
(n=78)\end{array}$} & RPR(beats/min) & $79.31 \pm 8.14$ & $77.15 \pm 6.24$ & $<0.01$ \\
\hline & $\mathrm{SBP}(\mathrm{mmHg})$ & $123.64 \pm 7.6$ & $121.03 \pm 6.51$ & $<0.01$ \\
\hline & $\mathrm{DBP}(\mathrm{mmHg})$ & $78.26 \pm 6.51$ & $76.64 \pm 4.96$ & $<0.01$ \\
\hline & fI $(\%)$ & $75.78 \pm 9.03$ & $77.30 \pm 7.14$ & $<0.01$ \\
\hline \multirow{4}{*}{$\begin{array}{l}\text { All } \\
(n=150)\end{array}$} & RPR(beats/min) & $77.39 \pm 7.22$ & $75.18 \pm 6.13$ & $<0.01$ \\
\hline & $\mathrm{SBP}(\mathrm{mmHg})$ & $120.33 \pm 8.81$ & $117.87 \pm 6.84$ & $<0.01$ \\
\hline & $\mathrm{DBP}(\mathrm{mmHg})$ & $77.13 \pm 5.65$ & $75.40 \pm 4.90$ & $<0.01$ \\
\hline & fI $(\%)$ & $72.97 \pm 10.98$ & $74.42 \pm 9.29$ & $<0.01$ \\
\hline
\end{tabular}

RPR - Resting Pulse Rate; SBP - Systolic Blood Pressure; DBP - Diastolic Blood Pressure; fI - Fitness Index

Table 4: Cardiovascular efficiency in control group (paired student's test)

\begin{tabular}{|c|c|c|c|c|}
\hline \multirow{2}{*}{ Gender } & \multirow{2}{*}{ Parameter } & Pre yoga & Post yoga & \multirow{2}{*}{$P$ value } \\
\hline & & $\operatorname{Mean} \pm$ SD & Mean \pm SD & \\
\hline \multirow{4}{*}{$\begin{array}{l}\text { Female } \\
(n=64)\end{array}$} & RPR(beats/min) & $76.16 \pm 5.35$ & $76.67 \pm 4.50$ & 0.11 \\
\hline & $\mathrm{SBP}(\mathrm{mmHg})$ & $119.66 \pm 8.24$ & $120.00 \pm 6.74$ & 0.75 \\
\hline & DBP(mmHg) & $77.25 \pm 4.21$ & $77.03 \pm 3.80$ & 0.77 \\
\hline & fI $(\%)$ & $71.93 \pm 11.38$ & $71.19 \pm 11.40$ & 0.96 \\
\hline \multirow{4}{*}{$\begin{array}{l}\text { Male } \\
(n=86)\end{array}$} & RPR(beats/min) & $76.29 \pm 4.64$ & $76.48 \pm 5.32$ & 0.80 \\
\hline & $\mathrm{SBP}(\mathrm{mmHg})$ & $120.95 \pm 6.54$ & $119.16 \pm 5.93$ & 0.07 \\
\hline & $\mathrm{DBP}(\mathrm{mmHg})$ & $76.77 \pm 4.91$ & $76.56 \pm 4.54$ & 0.78 \\
\hline & fI $(\%)$ & $77.05 \pm 7.32$ & $75.06 \pm 10.64$ & 0.12 \\
\hline \multirow{4}{*}{$\begin{array}{l}\text { All } \\
(n=150)\end{array}$} & RPR(beats/min) & $76.23 \pm 4.94$ & $76.56 \pm 4.97$ & 0.54 \\
\hline & $\mathrm{SBP}(\mathrm{mmHg})$ & $120.40 \pm 7.32$ & $119.52 \pm 6.28$ & 0.23 \\
\hline & $\mathrm{DBP}(\mathrm{mmHg})$ & $76.97 \pm 4.62$ & $76.76 \pm 4.23$ & 0.69 \\
\hline & fI $(\%)$ & $74.51 \pm 9.70$ & $73.41 \pm 11.09$ & 0.31 \\
\hline
\end{tabular}

Table 5: Respiratory function tests in intervention group (paired student's test)

\begin{tabular}{|c|c|c|c|c|}
\hline \multirow{2}{*}{ Gender } & \multirow{2}{*}{ Parameter } & Pre yoga & Post yoga & \multirow{2}{*}{ P value } \\
\hline & & Mean \pm SD & Mean \pm SD & \\
\hline \multirow{12}{*}{ Female $(n=72)$} & $\mathrm{TV}(\mathrm{ml})$ & $359.88 \pm 25.31$ & $361.83 \pm 25.40$ & 0.64 \\
\hline & SVC(liters) & $3.10 \pm 0.45$ & $3.47 \pm 0.57$ & $<0.01$ \\
\hline & BHT(seconds) & $37.22 \pm 5.06$ & $39.65 \pm 4.83$ & $<0.01$ \\
\hline & $\mathrm{MEP}(\mathrm{mmHg})$ & $64.11 \pm 11.24$ & $65.31 \pm 11.58$ & $<0.01$ \\
\hline & 40mmHg ET(seconds) & $28.04 \pm 9.57$ & $30.40 \pm 10.21$ & $<0.01$ \\
\hline & FEV1(liters) & $2.50 \pm 0.52$ & $2.62 \pm 0.71$ & $<0.01$ \\
\hline & FVC(liters) & $3.13 \pm 0.63$ & $3.25 \pm 0.85$ & $<0.01$ \\
\hline & FEV1/FVC(\%) & $79.86 \pm 2.98$ & $81.56 \pm 3.86$ & $<0.01$ \\
\hline & $\operatorname{PEFR}(\mathrm{L} / \mathrm{sec})$ & $382.72 \pm 26.63$ & $383.88 \pm 26.40$ & $<0.01$ \\
\hline & FEF $25-75 \%(\mathrm{~L} / \mathrm{sec})$ & $296.76 \pm 15.82$ & $297.29 \pm 15.75$ & $<0.01$ \\
\hline & FEF200-1200(L/sec) & $346.58 \pm 14.79$ & $347.11 \pm 14.83$ & $<0.01$ \\
\hline & MVV(L/sec) & $99.95 \pm 20.62$ & $100.03 \pm 20.60$ & $<0.01$ \\
\hline
\end{tabular}




\begin{tabular}{|c|c|c|c|c|}
\hline \multirow{2}{*}{ Gender } & \multirow{2}{*}{ Parameter } & Pre yoga & Post yoga & \multirow{2}{*}{ P value } \\
\hline & & Mean \pm SD & Mean \pm SD & \\
\hline \multirow{12}{*}{ Male $(n=78)$} & $\mathrm{TV}(\mathrm{ml})$ & $409.96 \pm 62.36$ & $412.92 \pm 62.41$ & 0.77 \\
\hline & SVC(liters) & $4.00 \pm 0.53$ & $4.30 \pm 0.50$ & $<0.01$ \\
\hline & BHT(seconds) & $41.77 \pm 9.08$ & $45.01 \pm 9.54$ & $<0.01$ \\
\hline & $\operatorname{MEP}(\mathrm{mmHg})$ & $74.95 \pm 19.26$ & $76.12 \pm 19.60$ & $<0.01$ \\
\hline & 40mmHg ET(seconds) & $34.68 \pm 13.07$ & $37.15 \pm 13.49$ & $<0.01$ \\
\hline & FEV1(liters) & $3.25 \pm 0.57$ & $3.46 \pm 0.59$ & $<0.01$ \\
\hline & FVC(liters) & $4.07 \pm 0.66$ & $4.30 \pm 0.71$ & $<0.01$ \\
\hline & FEV1/FVC (\%) & $79.91 \pm 3.29$ & $82.00 \pm 3.79$ & $<0.01$ \\
\hline & $\operatorname{PEFR}(\mathrm{L} / \mathrm{sec})$ & $419.73 \pm 48.67$ & $455.08 \pm 38.30$ & $<0.01$ \\
\hline & FEF25-75\% (L/sec) & $298.32 \pm 16.52$ & $298.88 \pm 16.48$ & $<0.01$ \\
\hline & FEF200-1200(L/sec) & $341.94 \pm 14.88$ & $342.46 \pm 14.89$ & $<0.01$ \\
\hline & MVV(L/sec) & $129.99 \pm 23.95$ & $130.42 \pm 23.94$ & $<0.01$ \\
\hline \multirow{12}{*}{ All $(n=150)$} & $\mathrm{TV}(\mathrm{ml})$ & $385.92 \pm 54.27$ & $388.40 \pm 54.55$ & 0.69 \\
\hline & SVC(liters) & $3.57 \pm 0.60$ & $3.90 \pm 0.68$ & $<0.01$ \\
\hline & BHT(seconds) & $39.59 \pm 7.74$ & $42.44 \pm 8.09$ & $<0.01$ \\
\hline & $\mathrm{MEP}(\mathrm{mmHg})$ & $69.75 \pm 16.78$ & $70.93 \pm 17.08$ & $<0.01$ \\
\hline & 40mmHg ET(seconds) & $31.49 \pm 11.96$ & $33.91 \pm 12.46$ & $<0.01$ \\
\hline & FEV1(liters) & $2.89 \pm 0.66$ & $3.06 \pm 0.81$ & $<0.01$ \\
\hline & FVC(liters) & $3.62 \pm 0.80$ & $3.80 \pm 0.94$ & $<0.01$ \\
\hline & FEV1/FVC (\%) & $79.89 \pm 3.13$ & $81.79 \pm 3.82$ & $<0.01$ \\
\hline & PEFR(L/sec) & $419.73 \pm 48.67$ & $420.90 \pm 48.62$ & $<0.01$ \\
\hline & FEF25-75\% (L/sec) & $294.57 \pm 16.15$ & $298.12 \pm 16.10$ & $<0.01$ \\
\hline & FEF200-1200(L/sec) & $344.17 \pm 14.97$ & $344.71 \pm 14.95$ & $<0.01$ \\
\hline & $\operatorname{MVV}(\mathrm{L} / \mathrm{sec})$ & $115.38 \pm 27.05$ & $115.84 \pm 27.03$ & $<0.01$ \\
\hline
\end{tabular}

Table 6: Respiratory function tests in control group (paired student's test)

\begin{tabular}{|c|c|c|c|c|}
\hline \multirow{2}{*}{ Gender } & \multirow{2}{*}{ Parameter } & Pre yoga & Post yoga & \multirow{2}{*}{$P$ value } \\
\hline & & Mean \pm SD & Mean \pm SD & \\
\hline \multirow{12}{*}{ Female $(n=64)$} & $\mathrm{TV}(\mathrm{ml})$ & $357.84 \pm 26.10$ & $358.67 \pm 25.74$ & 0.87 \\
\hline & SVC(liters) & $3.54 \pm 0.54$ & $3.55 \pm 0.59$ & 0.94 \\
\hline & BHT(seconds) & $37.27 \pm 5.33$ & $37.36 \pm 4.98$ & 0.88 \\
\hline & $\mathrm{MEP}(\mathrm{mmHg})$ & $62.91 \pm 11.06$ & $62.30 \pm 11.19$ & 0.10 \\
\hline & 40mmHg ET(seconds) & $27.05 \pm 9.72$ & $27.39 \pm 10.28$ & 0.35 \\
\hline & FEV1(liters) & $2.80 \pm 0.52$ & $2.75 \pm 0.60$ & 0.45 \\
\hline & FVC(liters) & $3.57 \pm 0.62$ & $3.52 \pm 0.77$ & 0.55 \\
\hline & FEV1/FVC (\%) & $78.34 \pm 3.33$ & $78.13 \pm 3.34$ & 0.71 \\
\hline & PEFR(L/sec) & $390.27 \pm 26.56$ & $390.31 \pm 26.61$ & 0.86 \\
\hline & FEF $25-75 \%(\mathrm{~L} / \mathrm{sec})$ & $302.10 \pm 16.61$ & $302.14 \pm 16.68$ & 0.92 \\
\hline & FEF200-1200(L/sec) & $345.81 \pm 15.00$ & $345.29 \pm 15.90$ & 0.21 \\
\hline & MVV(L/sec) & $99.95 \pm 20.39$ & $99.89 \pm 20.12$ & 0.88 \\
\hline
\end{tabular}




\begin{tabular}{|c|c|c|c|c|}
\hline \multirow{2}{*}{ Gender } & \multirow{2}{*}{ Parameter } & Pre yoga & Post yoga & \multirow{2}{*}{$P$ value } \\
\hline & & Mean \pm SD & Mean \pm SD & \\
\hline \multirow{12}{*}{ Male $(n=86)$} & $\mathrm{TV}(\mathrm{ml})$ & $395.08 \pm 48.07$ & $396.56 \pm 63.02$ & 0.86 \\
\hline & SVC(liters) & $3.76 \pm 0.64$ & $3.84 \pm 0.53$ & 0.32 \\
\hline & BHT(seconds) & $41.97 \pm 9.06$ & $42.29 \pm 9.13$ & 0.67 \\
\hline & $\mathrm{MEP}(\mathrm{mmHg})$ & $72.58 \pm 14.43$ & $73.45 \pm 18.86$ & 0.46 \\
\hline & 40mmHg ET(seconds) & $35.20 \pm 12.86$ & $34.95 \pm 13.32$ & 0.68 \\
\hline & FEV1(liters) & $2.97 \pm 0.65$ & $3.02 \pm 0.63$ & 0.65 \\
\hline & FVC(liters) & $3.72 \pm 0.58$ & $3.81 \pm 0.76$ & 0.44 \\
\hline & FEV1/FVC (\%) & $79.93 \pm 3.31$ & $79.06 \pm 3.27$ & 0.10 \\
\hline & PEFR(L/sec) & $393.34 \pm 36.12$ & $392.85 \pm 35.96$ & 0.12 \\
\hline & FEF25-75\% (L/sec) & $306.48 \pm 16.56$ & $309.44 \pm 16.08$ & 0.17 \\
\hline & FEF200-1200(L/sec) & $349.66 \pm 15.70$ & $350.00 \pm 15.18$ & 0.49 \\
\hline & MVV(L/sec) & $101.90 \pm 23.83$ & $102.42 \pm 23.81$ & 0.88 \\
\hline \multirow{12}{*}{ All $(n=150)$} & $\mathrm{TV}(\mathrm{ml})$ & $379.19 \pm 44.13$ & $380.39 \pm 53.84$ & 0.82 \\
\hline & SVC(liters) & $3.67 \pm 0.61$ & $3.72 \pm 0.57$ & 0.43 \\
\hline & BHT(seconds) & $39.96 \pm 8.02$ & $40.19 \pm 8.00$ & 0.78 \\
\hline & $\mathrm{MEP}(\mathrm{mmHg})$ & $68.45 \pm 13.91$ & $68.69 \pm 16.93$ & 0.64 \\
\hline & 40mmHg ET(seconds) & $31.72 \pm 12.27$ & $31.73 \pm 12.65$ & 0.98 \\
\hline & FEV1(liters) & $2.90 \pm 0.60$ & $2.90 \pm 0.63$ & 0.96 \\
\hline & FVC(liters) & $3.65 \pm 0.72$ & $3.69 \pm 0.78$ & 0.67 \\
\hline & FEV1/FVC (\%) & $79.25 \pm 3.40$ & $78.66 \pm 3.32$ & 0.13 \\
\hline & $\operatorname{PEFR}(\mathrm{L} / \mathrm{sec})$ & $392.03 \pm 32.32$ & $391.77 \pm 32.23$ & 0.22 \\
\hline & FEF25-75\% (L/sec) & $304.61 \pm 16.67$ & $306.32 \pm 16.68$ & 0.17 \\
\hline & FEF200-1200(L/sec) & $348.02 \pm 15.48$ & $347.99 \pm 15.61$ & 0.94 \\
\hline & MVV(L/sec) & $101.07 \pm 22.37$ & $101.34 \pm 22.28$ & 0.89 \\
\hline
\end{tabular}

\section{Discussion}

Cardiovascular efficiency parameters: In our study RPR, SBP and DBP reduced significantly whereas Fitness Index (FI) increased significantly following yoga training in participant students compared to control group. The similar effect oncardiovascular parameters was found by Bhavanani et al wherein participants practiced pranayama such as sukhapranayasms and Chandra nadi pranayama ${ }^{(10,11)}$. Many studies have reported that practice of pranayams increases parasympathetic activity and decreases sympathetic activity which may be responsible for these observed cardiac parameters $^{(12)(13)(14)}$.

The calculated rateofbreathing in different pranayams may have contributed to the beneficial cardiovascular effects seen in this study; previous studies have reported that slow and deep breathing decreases the heart rate and heart rate variability possibly due to enhanced vagal tone on sinoatrial node ${ }^{(15)}$. Such enhanced vagal modulation may be responsible for decreased heart rate and blood pressure observed following yoga ${ }^{(16)}$. Bernardi et al reported that slow and deep breathing combines $R R$ interval fluctuations with the rate of respiration and significantly increases their amplitude and thereby enhances baro-reflex competence ${ }^{(17)}$, such enhancement may have contributed to the lowering of blood pressure needs to be reduced. Thus yoga may serve as a simple cost effective adjunct in the management of hypertension in addition to the regular antihypertensive management. Jasmin et al found effect of yoga in diabetic patient to reduce pain with diabetic medication ${ }^{(4)}$.

The RPR and DBP were significantly lower post 
yoga in our study. A similar decrease in RPR and DBP was reported by Bhavani et al. and Bhutkaretal ${ }^{(18)(19)}$. The main determining factor of DBP is peripheral vascular resistance which is regulated by sympathetic tone ${ }^{(20)}$. In the present study significant decrease in DBP and RPR in yoga group may be attributed to a decrease in peripheral vascular resistance due to reduced sympathetic tone. The SBP was found to be significantly lower in yoga group after training; Bhutkar et al also reported a similar decrease in $\mathrm{SBP}^{(19)}$. Interestingly Bhavanani $\mathrm{AB}$ et al reported an increase in SBP following fast Suryanamaskar practice which may be due to the speed of Suryanamaskar causing increasing venous return and cardiac output which resembles aerobic exercise ${ }^{(18)(21)}$.

The observed cardiovascular changes may also be due to the shavasan which is reported to reduce cardiac sympathetic modulation ${ }^{(22)}$. Yoga practices including relaxation (such as shavasan) improves autonomic modulation and enhances vagal dominance. Hence shavasan may be included in the non pharmacological management of hypertension.

Respiratory efficiency parameter: We found a significant increase in VC, BHT,MEP and $40 \mathrm{mmHg}$ ET following yoga. While the change in TV was not statistically significant,similar results were reported by karmur et al and mahajan et al. yoga postures (asnas) such as suryanamaskar in our study increase skeletal muscle strength including those respiratory muscles ${ }^{(13,23)(24)}$. Hence lungs and thorax expand and collapse to the fullest possible. Such strengthening of the respiratory musculature contributes to the increased respiratory efficiency evidenced in our body.

Pulmonary Function Tests: Lung functions depend on many factors including neuropsychological factors and strength of respiratory muscles. We found that all the parameters i.e. FEV1,FVC, FEV1/FVC,PEFR, FEF25-75\%, FEF200-1200ml and MVV increased significantly after 4 weeks of yoga in healthy young adults'subjects, irrespective of the gender. Similar results were reported by various previous studies $(18,19,23,25)$. During yoga training especially during pranayama there are maximal inflation and deflation of the lungs which cause increased strength recruitment and endurance of respiratory musculature. Such maximal inflation and deflation act as a physiological stimulus causing secretion of prostaglandins and surfactants in the alveoli which thereby increase the lung compliance ${ }^{(26,27)}$. There is a reflex increases airway diameter and decreases resistance to air flow which causes the dynamic PFTs to improve. All the participants were investigated by the same investigators under similar conditions.

\section{Conclusion}

Yoga can be advocated as an adjunctive or alternative to conventional therapy for cardio respiratory disorders. We encourage yoga practice as physical activity during undergraduate curriculum in medical study to improve heath condition of medical students.

\section{Conflict of Interest: No}

Source of Funding: Self

Ethical Clearance: Yes

\section{References}

1. Pal G. Yoga and heart rate variability. Int J Clin Exp Physiol. 2015;2(1):2.

2. Collins C. Yoga: Intuition Preventive Medicine Treatment. J Obstet Gynecol Neonatal Nurs [Internet]. 1998 Sep [cited 2020 Feb 2];27(5):5638.

3. Solari E. Longitudinal prediction of 1st and 2nd grade English oral reading fluency in ELL. J Adolesc [Internet]. 2014;74(4):274-83.

4. Parmar J, Kagathara JD, Mistry AH, Patel VJ. A Randomized Control Study on Effect of 12 Weeks Yoga Therapy in Type 2 Diabetes Mellitus Patients with Distal Polyneuropathy. Int Physiol [Internet]. 2017;5(2):77-82.

5. Schmid AA, Van Puymbroeck M, Altenburger PA, Schalk NL, Dierks TA, Miller KK, et al. Poststroke balance improves with yoga: A pilot study. Stroke. 2012;

6. Telles S, Gaur V, Balkrishna A. Effect of a yoga practice session and a yoga theory session on state anxiety. Percept Mot Skills. 2009 Dec;109(3):924 30.

7. Salmon P, Lush E, Jablonski M, Sephton SE. Yoga and Mindfulness: Clinical Aspects of an Ancient Mind/Body Practice. Cogn Behav Pract. 2009 Feb;16(1):59-72.

8. Stueck M, Gloeckner N. Yoga for children in the mirror of the science: Working spectrum and practice fields of the Training of Relaxation with Elements of Yoga for Children. Early Child Dev Care. 2005;175(4):371-7. 
9. Gallagher JR, Brouha L. V. A Simple Method of Evaluating Fitness in Boys: The Step Test. Yale J Biol Med [Internet]. 1943 Jul [cited $2020 \mathrm{Feb}$ 6];15(6):769-79. Available from: http://www.ncbi. nlm.nih.gov/pubmed/21434109

10. Bhavanani AB, Sanjay Z, Madanmohan. Immediate effect of sukha pranayama on cardiovascular variables in patients of hypertension. Int J Yoga Therap. 2011;(21):73-6.

11. Bhavanani A, Sanjay Z, Madanmohan. Immediate effect of chandra nadi pranayama (left unilateral forced nostril breathing) on cardiovascular parameters in hypertensive patients. Int $\mathrm{J}$ Yoga. 2012;5(2):108.

12. Chu IH, Wu WL, Lin IM, Chang YK, Lin YJ, Yang PC. Effects of Yoga on Heart Rate Variability and Depressive Symptoms in Women: A Randomized Controlled Trial. J Altern Complement Med [Internet]. 2017.

13. Ashok S, Aparna P. Yoga As an Exercise Tool: Study of Physiological Parameters of the Healthy Subjects. Orig Artic Int J Basic Appl Physiol Int J Basic Appl Physiol. 2016;5(1):2016.

14. MuralikrishnanK,BalakrishnanB,Balasubramanian K, Visnegarawla F. Measurement of the effect of Isha Yoga on cardiac autonomic nervous system using short-term heart rate variability. J Ayurveda Integr Med. 2012 Apr 1;3(2):91-6.

15. Sroufe LA. EFFECTS OF DEPTH AND RATE OF BREATHING ON HEART RATE AND HEART RATE VARIABILITY. Psychophysiology [Internet]. 1971 Sep [cited 2020 Feb 2];8(5):64855.

16. Wang SZ, Li S, Xu XY, Lin GP, Shao L, Zhao $\mathrm{Y}$, et al. Effect of slow abdominal breathing combined with biofeedback on blood pressure and heart rate variability in prehypertension. J Altern Complement Med [Internet]. 2010 Oct [cited 2020 Feb 2];16(10):1039-45.

17. Bernardi L, Sleight P, Bandinelli G, Cencetti S, Fattorini L, Wdowczyc-Szulc J, et al. Effect of rosary prayer and yoga mantras on autonomic cardiovascular rhythms: Comparative study. $\mathrm{Br}$ Med J. 2001 Dec 22;323(7327):1446-9.
18. Jatiya L, Udupa $\mathrm{K}$, Balayogi Bhavanani A. EFFECT OF YOGA TRAINING ON HANDGRIP, RESPIRATORY PRESSURES AND PULMONARY FUNCTION. Vol. 47, Indian J Physiol Pharmacol. 2003.

19. Bhutkar PM, Bhutkar M V, Taware GB. Effect of Suryanamaskar Practice on Cardio-respiratory Fitness Parameters : A Pilot Study . Al Ameen J Med Sci [Internet]. 2008;1(2):126-9.

20. Hilton SM, Spyer KM. Central Nervous Regulation of Vascular Resistance. Annu Rev Physiol [Internet]. 1980 Oct [cited 2020 Feb 2];42(1):399411.

21. Bhavanani A, Madanmohan, Udupa K, Ravindra P. A comparative study of slow and fast suryanamaskar on physiological function. Int J Yoga. 2011;4(2):71.

22. Santaella DF, Geraldo Lorenzi-Filho I, Marcos Rodrigues IR, Taís Tinucci I, Ana Paula Malinauskas I, Dé cio Mion-Jú nior I, et al. Yoga Relaxation (savasana) decreases cardiac sympathovagal balance in hypertensive patients. MEDICALEXPRESS. 2014;1(5):233-8.

23. Karmur keshur A, Joshi VS, Padalia MS, Sarvaiya JL. Effect of ten weeks yoga practice on pulmonary function tests. Int J Biomed Adv Res J [Internet]. 2015 [cited 2020 Feb 2];6(9):682-5.

24. Naveen Kumar M, Thanalakshmi J, Kannan R, Mahesh Kumar K, Allu AR, Vijayalakshmi B. The immediate effect of sheethali and sheethkari pranayama on blood pressure and cardiovascular changes among hypertensive patients. Int $\mathrm{J}$ Res Pharm Sci. 2018;9(4):1249-52.

25. Sodhi C, Singh S, Dandona PK. A STUDY OF THE EFFECT OF YOGA TRAINING ON PULMONARY FUNCTIONS IN PATIENTS WITH BRONCHIAL ASTHMA.

26. Hooper SB, Wallace MJ. Physical, Endocrine and Growth Factors in Lung Development. In: The Lung: Development, Aging and The Environment. Elsevier Inc.; 2004. p. 131-48.

27. Orgeig S, Daniels CB, Sullivan LC. Development of the Pulmonary Surfactant System. Lung Dev Aging Environ. 2003 Dec 3;149-67. 